\title{
Dependence of plastic properties of metals on the density of energy absorbed during deformation
}

\author{
V.M. Kosenkov \\ v.m.kosenkov@gmail.com \\ Institute of Pulse Processes and Technologies NAS of Ukraine, Oktyabr'skiy Ave., 43a, 54018 Nikolayev,. Ukraine
}

Energy consumption of cold plastic deforming the materials determines the efficiency of technological methods and their competitiveness. Specific energy consumption of deforming the materials used often to analyze these processes should be determined for scientific and practical interest. This subject is covered in the articles little. Therefore, the main objective of the present research was to investigate the plastic deformation of metals depending on the density of the energy absorbed during the process of their cold deforming performed in three different stretching ways: quasi-static, shock-impact and pulse electrohydraulic. Specimens of 6111, BH240 and DP780 alloys were stretched quasi-static at deforming rates in the range 0.1 to $0.3 \mathrm{~s}^{-1}$. Work of stretching and the density of the energy absorbed within the deformed section of a sample depending on the plastic deformation were obtained from the diagrams. Shock-impact stretching of these same alloys was performed at rates of 200 to $2000 \mathrm{~s}^{-1}$ using the method of Kolskiy and a Hopkinson dissected bar. In this study a scheme of stretching flat samples without stretching a Hopkinson bar was worked out. Pulse electrohydraulic method was used for biaxial deforming the plates. The amount of energy released in a discharge channel, the energy of deforming the specimens and their own deformations equivalent to uniaxial stretching were determined. It was found out as a result of the research that the average deformation on the volume of the plate equivalent to uniaxial stretching the alloys is independent actually on the parameters of pulse electrohydraulic deforming. But the density of the energy absorbed within the alloy during deforming, a deforming rate and a type of the alloys have crucial influence on it. High speed deforming of the high hard steels at rates up to $1000 \mathrm{~s}^{-1}$ requires $25 \%$ as much energy as static deforming. The geometrical characteristics of the discharge chamber and rigging affect the efficiency of the process of deforming the sheet metal alloys significantly.

Keywords: plasticity, tensile impact, electrical discharge in water, sheet high-strength alloys, velocity of plastic deformation.

\section{Зависимость пластических свойств металлов от плотности энергии, поглощённой в процессе деформирования}

\author{
Косенков В.M. \\ v.m.kosenkov@gmail.com
}

Институт импульсных процессов и технологий НАН Украины, пр. Октябрьский, 43а, 54018, Николаев, Украина

Затраты энергии в процессе холодного пластического деформирования материалов определяют эффективность технологических методов и их конкурентоспособность. Для анализа этих процессов используют удельные затраты энергии деформирования материалов, определение которых актуально в научном и практическом отношении. Эти сведения мало освещены в публикациях. Поэтому основной целью данной работы было исследование зависимости пластических деформаций металлов от плотности энергии, поглощённой в процессе их холодного деформирования тремя разными способами: квазистатическим, ударного растяжения и импульсным электрогидравлическим. Квазистатическое растяжение образцов сплавов 6111, BH240 и DP780 выполняли со скоростью деформации от 0,1 до 0,3 $\mathrm{c}^{-1}$. По полученным диаграммам определяли работу растяжения и плотность энергии, поглощённой деформируемой частью образцов в зависимости от пластической деформации. Ударное растяжение образцов указанных сплавов со скоростью деформации от 200 до $2000 \mathrm{c}^{-1}$ выполняли по методу Кольского с использованием разрезного стержня Гопкинсона. В данном исследовании разработана схема растяжения плоских образцов без растяжения стержня Гопкинсона. Импульсный электрогидравлический способ применяли для двухосного деформирования пластин. Определяли количество энергии, выделившееся в канале разряда, энергию деформирования образцов и их деформацию, эквивалентную одноосному растяжению материала. В результате выполненных исследований определено, что сред- 
няя деформация по объёму пластины, эквивалентная одноосному растяжению исследованных сплавов практически не зависит от параметров импульсного электрогидравлического деформирования, а определяющее влияние на неё оказывают: плотность энергии, поглощенной сплавом в процессе деформирования, скорость деформации и вид сплава. Высокоскоростное деформирование высокопрочных сталей при скоростях деформации до $1000 \mathrm{c}^{-1}$ требует до $25 \%$ больших затрат энергии, чем статическое деформирование. Геометрические характеристики разрядной камеры и оснастки существенно влияют на КПД процесса деформирования листовых сплавов.

Ключевые слова: пластичность, ударное растяжение, электрический разряд в воде, листовые высокопрочные сплавы, скорость пластической деформации.

\section{1. Введение}

Затраты энергии в процессе холодного пластического деформирования материалов в большинстве случаев определяют эффективность технологических методов и их конкурентоспособность. Большое разнообразие этих методов затрудняет выполнение сравнительного анализа их использования, тем более что механические характеристики (статические и динамические) современных материалов также существенно отличаются друг от друга $[1,2]$. Первичной (ключевой) информацией для анализа этих процессов служат удельные затраты энергии деформирования материалов в зависимости от используемого метода, определение которых актуально в научном и практическом отношении.

Эти сведения мало освещены в публикациях. Зависимости плотности энергии деформирования металлов от пластической деформации приведены в работах $[3,4]$ для технически чистой меди и легированной стали марки HSLA в условиях термомеханического деформирования. Очень краткие данные об этом приведены в $[5,6]$. Информация о влиянии способов холодного пластического деформирования материалов на плотность энергии деформирования очень ограничена [7]. Поэтому основной целью данной работы было исследование зависимости пластических деформаций металлов от плотности энергии, поглощённой в процессе их холодного деформирования тремя существенно разными способами, такими как квазистатический, ударного растяжения и импульсный электрогидравлический.

\section{2. Методика исследований}

Квазистатическое растяжение образцов сплавов 6111, BH240 и DP780 (толщиной $\delta 1,4,0,65$ и 1,35 мм, соответственно) выполняли на разрывной машине со скоростью деформации от 0,1 до $0,3 \mathrm{c}^{-1}$. Образцы изготавливали из листового материала (рис.1). По полученным диаграммам определяли работу растяжения и плотность энергии, поглощённой деформируемой частью образцов в зависимости от пластической деформации.

Ударное растяжение образцов указанных сплавов со скоростью деформации от 200 до $2000 \mathrm{c}^{-1}$ выполняли по методу Кольского с использованием разрезного стержня Гопкинсона $[8,9]$. Этот метод позволяет в процессе нагружения образцов получать диаграммы динамического деформирования материалов с известной скоростью деформации.
Схема установки Гопкинсона-Кольского, метод измерения и обработки полученных данных для определения диаграмм деформирования сплавов подробно описаны в работах [9-11]. Интегрируя полученные диаграммы, определяли работу растяжения материала, разделив которую на объём деформируемой части образца, вычисляли плотность энергии деформирования.

Существующие методы испытания материалов на растяжение используют модификации метода Гопкинсона-Кольского [12,13], отличающиеся от классического метода способом ударного нагружения образца, приводящего к его растяжению. Растяжение образца происходит, когда стержни Гопкинсона также испытывают растяжение. В данном исследовании разработана схема растяжения плоских образцов (рис.2) без растяжения стержней Гопкинсона.

Согласно этой схеме, один конец образца 1 крепится к нагружающему стержню 2, а второй - к опорному стержню 3 с помощью болтов 4 (рис. 3). Для предотвращения разрушения стержней при их соударении после разрыва образца установлены резиновые демпферы 5. Форма и размеры образцов (рис.1,2) соответствуют требованиям ГОСТ $1497-84$ (ИСО 6892-84) для испыта-

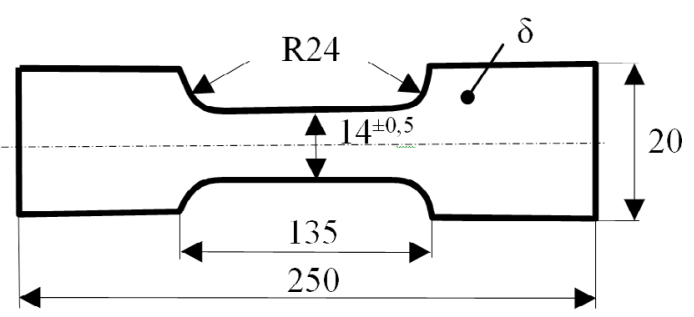

Рис. 1. Образец для квазистатического растяжения листового материала.

Fig. 1. Model for quasi-static stretching of sheet material.

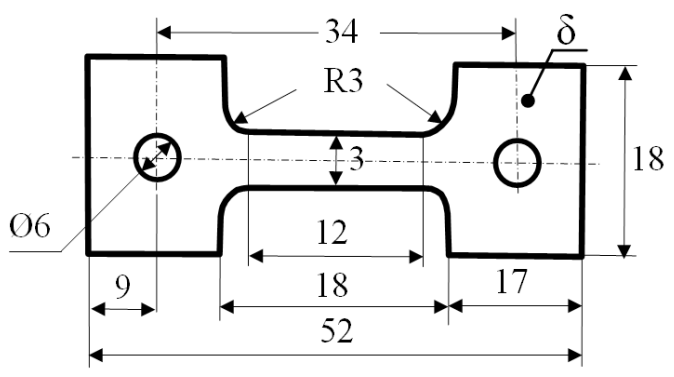

Рис. 2. Образец для ударного растяжения листового материала. Fig. 2. The sample for shock stretching of sheet material. 
ния металлов на растяжение. Использование такой схемы растяжения образцов (рис. 3) не вызывает появления дополнительных искажений сигнала отражёнными волнами, а также не приводит к изменению метода обработки данных [9-11].

Для регистрации сигналов от тензорезисторов использовали цифровой запоминающий осциллограф Tektronix TDS2012B. Обработку данных выполняли с помощью программы MS Excel. Погрешность определения напряжений и деформаций по разработанной схеме нагружения образцов лежит в пределах $\pm 5 \%$.

Импульсный электрогидравлический способ двухосного деформирования образцов осуществляли по схеме, показанной на рисунке 4.

Экспериментальное устройство позволяло изменять расстояние между электродами $\left(l_{\mathrm{ch}}\right)$, расстояние от оси

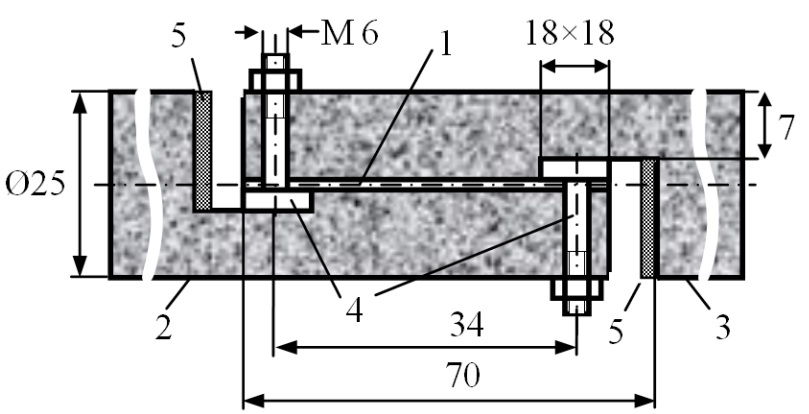

Рис. 3. Схема крепления плоского образца.

Fig. 3. The scheme of fastening of a flat sample.

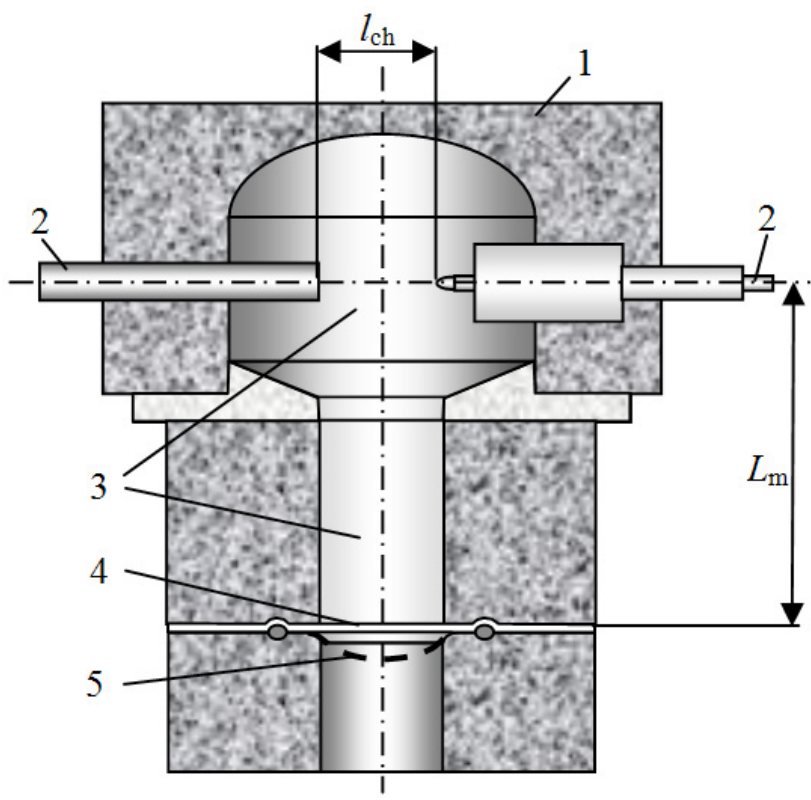

Рис. 4. Схема нагружения пластины. 1 - корпус разрядной камеры; 2 - электроды; 3 - полости разрядной камеры и оснастки, заполненные водой; 4 - пластина до деформации; 5 - профиль деформированной пластины; $l_{\text {ch }}-$ расстояние между электродами; $L_{\mathrm{m}}-$ расстояние от оси электродов до поверхности пластины.

Fig. 4. Scheme of plate loading. 1 - body of the discharge chamber; 2 - electrodes; 3 - cavity of the discharge chamber and tooling filled with water; 4 - plate before deformation; $5-$ profile of the deformed plate; $l_{\mathrm{ch}}$ is the distance between electrodes; $L_{\mathrm{m}}$ is the distance from the axis of the electrodes to the plate surface. электродов до поверхности образца $\left(L_{\mathrm{m}}\right)$, радиус отверстия матрицы $(R)$ и радиус галтели $(R)$. В процессе исследования варьировали электрические параметры разрядного контура: ёмкость $(C)$ и напряжение заряда конденсаторной батареи $\left(U_{0}\right)$, а также его индуктивность $(L)$. В процессе разряда выполняли запись осциллограмм силы тока и напряжения между электродами, что позволило определять количество энергии, выделившееся в канале разряда. Энергию деформирования образцов и их деформацию, эквивалентную одноосному растяжению материала, определяли по методу [14]. Отношение энергии деформирования к объёму деформируемой части образца позволяло вычислить плотность энергии его деформирования.

В процессе исследования зависимости пластических деформаций пластины от плотности поглощённой ею энергии варьировали $U_{0}$ от 30 до 45 кВ и $l_{\text {ch }}$ от 20 до 82 мм при неизменной ёмкости конденсаторной батареи $(C=34$ мкФ) и индуктивности разрядного контура $(L=6,1$ мкГн). B этом диапазоне изменения параметров энергия заряда конденсаторной батареи изменялась от 14 до 34 кДж. Удельная электропроводность воды при $23^{\circ} \mathrm{C}$ составляла 0,045 См/м. Активное сопротивление элементов разрядного контура, за исключением канала разряда, равно 0,04 Ом. Расстояние $L_{\mathrm{m}}$ изменяли от 170 до 320 мм, радиус отверстия матрицы - от 20 до 50 мм, радиус галтели - от 2 до 8 мм. Использовали пластины сплавов ВН240 ( $\delta=0,65$ мм), DP780 ( $\delta=1,35$ мм) и 6111 ( $\delta=1,4$ мм). Для контроля распределения деформаций в пластинах на их поверхности наносили квадратную контрольную сетку. Если на выбранном режиме разряда пластины деформировались до разрушения, тогда использовали пакет из двух или трех пластин. Существенного расслоения пакета пластин в процессе их деформирования не наблюдалось, что свидетельствовало об эквивалентности замены одной пластины пакетом пластин. В этом случае считали, что толщина эквивалентной пластины равна толщине пакета пластин. Прогибы пластин в пакете отличались друг от друга только в пределах погрешности их измерения ( $\pm 0,1$ мм). Средняя скорость деформации пластин не превышала $1000 \mathrm{c}^{-1}$.

\section{3. Результаты исследования}

Из трёх использованных в данной работе методов деформирования сплавов наибольшей сложностью реализации отличается метод импульсного электрогидравлического деформирования пластин. Несмотря на такой недостаток, отмечено существенное (до 2,5 раз) увеличение предельных пластических деформаций материала в процессе двухосного деформирования пластин этим способом [7], что свидетельствует о целесообразности его использования.

Энергия деформирования пластины импульсным электрогидравлическим способом зависит не столько от энергии, запасаемой в конденсаторной батарее, сколько от энергии, выделившейся в канале разряда. Поэтому в результате обработки осциллограмм определили зависимость электрического КПД разряда (отношение энер- 
гии, выделившейся в канале разряда, к энергии заряда конденсаторной батареи) от расстояния между электродами (рис. 5). Как следует из рисунка 5, для выбранных параметров разрядного контура, максимальный электрический КПД разряда $\left(\approx 80 \%\right.$, когда $U_{0}=45$ кВ) достигается при расстоянии между электродами приблизительно равном 90 мм. При этом статистический разброс данных, вследствие нестабильности пробоя воды, составлял $\pm 8 \%$.

По измеренному прогибу пластин с помощью метода [14] определили энергию и КПД процесса их деформирования при $U_{0}=45 \mathrm{\kappa}, \mathrm{R}=20$ мм (рис. 6). Из данных на рисунке 6 следует, что механический КПД процесса деформирования существенно зависит от расстояния между электродами. Увеличение $l_{\text {ch }}$ от 20 до 82 мм приводит к возрастанию КПД процесса деформирования от 1,7 до 4 раз. При этом электрический КПД разряда увеличивается только в 1,6 раза (рис. 5,6 ). В целом, КПД процесса деформирования $\left(\eta_{\mathrm{p}}\right)$ при заданных параме-

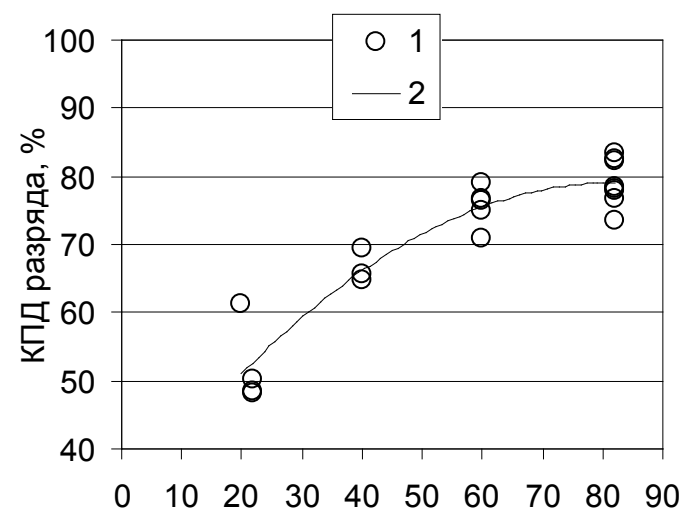

Расстояние между электродами, мм

Рис. 5. Электрический КПД разряда. 1 - эксперимент; 2 аппроксимация.

Fig. 5. Electrical efficiency of the discharge. 1 - experiment; 2 approximation.

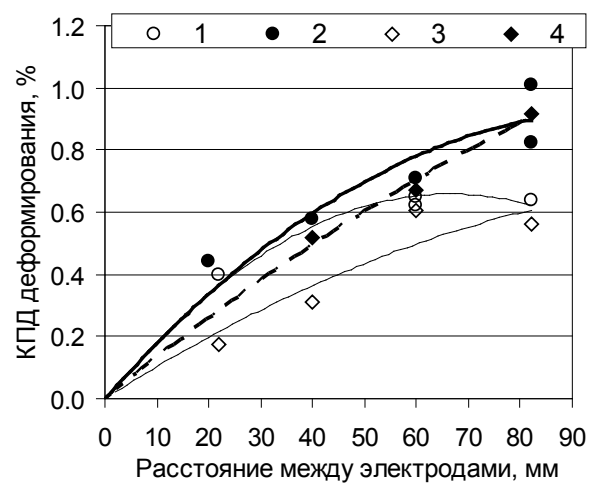

Рис. 6. КПД деформирования пластин. 1 - сплав 6111, $L_{\mathrm{m}}=180$ мм; 2 - сплав 6111, $L=273$ мм; 3 - сплав ВН240, $L=180$ мм; 4 - сплав $\mathrm{BH} 240, L_{\mathrm{m}}=273$ мм; 6111 - сплошные линии; $\mathrm{BH} 240$ - штриховые линии; $L_{\mathrm{m}}=180$ мм - тонкие линии; $L_{\mathrm{m}}=273$ мм - жирные линии.

Fig. 6. The efficiency of plates deformation. 1 - alloy $6111, L_{\mathrm{m}}=180 \mathrm{~mm}$; 2 - alloy 6111, $L_{\mathrm{m}}=273 \mathrm{~mm} ; 3$ - alloy BH240, $L_{\mathrm{m}}=180 \mathrm{~mm} ; 4$ - alloy $\mathrm{BH} 240, L_{\mathrm{m}}=273 \mathrm{~mm} ; 6111$ - solid lines; $\mathrm{BH} 240$ - dashed lines; $L_{\mathrm{m}}=180 \mathrm{~mm}$ - thin lines; $L_{\mathrm{m}}=273 \mathrm{~mm}$ - bold lines. трах системы очень мал. При рассмотренных параметрах нагружения и деформирования он в основном меньше $1 \%$. Существенное влияние на $\eta_{\text {p }}$ оказывает радиус отверстия матрицы $(R)$. Его увеличение от 20 до 50 мм приводит к возрастанию $\eta_{\text {p }}$ приблизительно в 10 раз, даже при малых $l_{\mathrm{ch}}=20$ мм.

Отмечено существенное влияние формы и размеров разрядной камеры на величину $\eta_{p}$. В частности, увеличение расстояния от оси электродов до пластины $\left(L_{\mathrm{m}}\right)$ от 180 до 273 мм за счёт удлинения отверстия оснастки

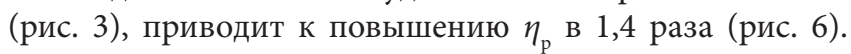
Этот эффект можно объяснить явлением «фокусировки» волн давления за счёт их отражения от стенок отверстия оснастки на пути к пластине. Это подтверждается увеличением давления на поверхность пластин $\left(P_{\mathrm{m}}\right)$ в конце их пластического деформирования [14]. Изменение $\left(L_{\mathrm{m}}\right)$ от 180 до 273 мм увеличивает давление $P_{\mathrm{m}}$ в пределах от 1,2 до 1,4 раза. Величина $P_{\mathrm{m}}$, также как и $\eta_{\mathrm{p}}$, существенно зависит от $l_{\mathrm{ch}}$, увеличиваясь от 2 до 4 раз при возрастании $l_{\mathrm{ch}}$ от 20 до 82 мм.

Величины $\eta_{p}$ и $P_{m}$ увеличиваются в пределах от 2 до 4 раз при возрастании электрического КПД разряда в 1,6 раза.

Сравнение деформаций пластин, полученных в процессе одноосного и двухосного нагружения разными способами возможно, если использовать понятие эквивалентной деформации материала. Деформация элементарного объема пластины, эквивалентная одноосному растяжению материала (ع), зависит от нормальных компонент тензора деформаций и определяется по формуле $[15,16]$

$$
\varepsilon=\sqrt{2\left(\varepsilon_{r}^{2}+\varepsilon_{\theta}^{2}+\varepsilon_{z}^{2}\right) / 3},
$$

где $\varepsilon_{\mathrm{r}}-$ радиальная деформация; $\varepsilon_{\theta}-$ окружная деформация; $\varepsilon_{z}-$ деформация по толщине пластины.

Осреднение $\varepsilon$ по всему объёму деформируемой части пластины позволяет определить среднюю деформацию по объёму пластины, эквивалентную одноосному растяжению материала $\left(\varepsilon^{*}\right)$.

Зависимости $\varepsilon^{\star}$ от плотности энергии деформирования $\left(E^{*}\right)$ представлены на рисунке 7. Зависимости $\varepsilon^{*}$ от $E^{\star}$, в рассмотренной области изменения параметров системы, близки к линейным.

Характерно, что линейные зависимости $\varepsilon^{*}$ от $E^{*}$ для каждого вида материала не изменяются, если в этой системе координат представить все данные, полученные в результате исследования, отличающиеся параметрами разрядного контура $\left(C, U_{0}, l_{\mathrm{ch}}\right)$ и геометрическими характеристиками $\left(L_{\mathrm{m}}, R, R\right.$ ) (рис. 7). По-видимому, эти зависимости можно считать энергетическими характеристиками определённого материала. Подобные зависимости отмечены в публикациях $[3,4]$ для технически чистой меди и легированной стали. На рисунке 7 также показаны характеристики, полученные в результате квазистатического (сплошные линии) и ударного (скорость деформации от 700 до $1000 \mathrm{c}^{-1}$ ) растяжения (штриховые линии) сплавов 6111, ВН240 и DP780 в процессе их одноосного нагружения. Как следует из рисунка 7, данные, полученные в результате одноосного и двухосного нагружения сплавов, хорошо согласуются друг с другом. 


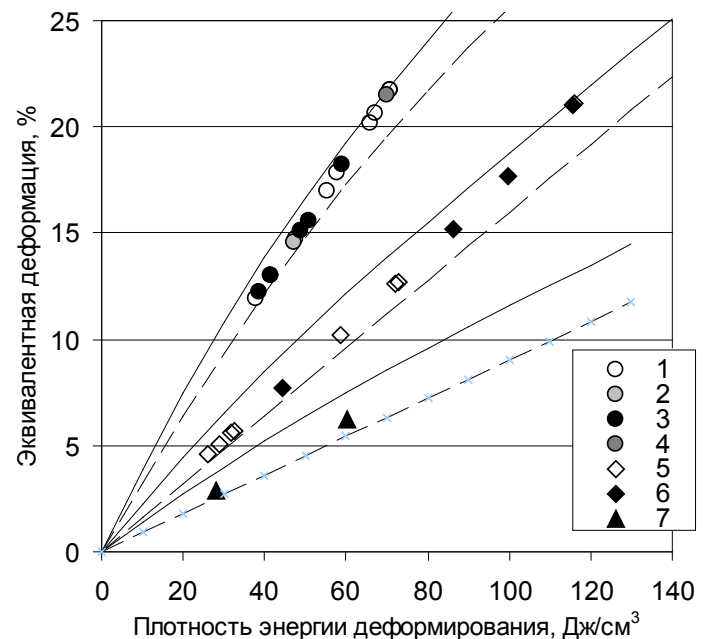

Рис. 7. Зависимости средней деформации по объёму пластины, эквивалентной одноосному растяжению пластин от плотности энергии их деформирования разными способами. 1-4 $6111 ; 5$ и 6 - BH240; 7 - DP780; точки - импульсное электрогидравлическое деформирование сплавов со средней скоростью деформации не более $1000 \mathrm{c}^{-1}$; сплошные линии квазистатическое растяжение; штриховые линии - ударное растяжение сплавов со скоростью деформации от 700 до $1000 \mathrm{c}^{-1}$.

Fig. 7. Dependences of the average deformation across the plate volume which is equivalent to uniaxial plate stretching and the energy density of their deformation by different ways. 1 - 4 - 6111; 5 and 6 - BH240; 7 - DP780; points - pulse electrohydraulic deformation of alloys with an average strain rate of $1000 \mathrm{~s}^{-1}$; solid lines - quasi-static stretching; dashed lines - shock stretching of alloys with a strain rate from 700 to $1000 \mathrm{~s}^{-1}$.

\section{4. Заключение}

По результатам выполненных исследований пластического деформирования высокопрочных сплавов можно сделать следующие основные выводы:

- средняя деформация по объёму пластины, эквивалентная одноосному растяжению исследованных сплавов практически не зависит от параметров импульсного электрогидравлического деформирования, а определяющее влияние на неё оказывают: плотность энергии, поглощенной сплавом в процессе деформирования, скорость деформации и вид сплава;

- высокоскоростное деформирование высокопрочных сталей при скоростях деформации до $1000 \mathrm{c}^{-1}$ требует до $25 \%$ больших затрат энергии, чем статическое деформирование;

- геометрические характеристики разрядной камеры и оснастки существенно влияют на КПД процесса деформирования листовых сплавов.

\section{Литература / References}

1. High-Strength Steels. American Technical Publishers Ltd. - USA: Prd 800 Engineering Metallic Materials. (2012) $16 \mathrm{p}$.

2. International Alloy Designations and Chemical Composition Limits for Wrought Aluminum and Wrought Aluminum Alloys. The Aluminum Association, Inc., Wilson Boulevard, Arlington, (2009) $37 \mathrm{p}$.

3. J.D. Clayton, D.L. McDowell. International Journal of Plasticity. 19(9) P. 1401-1444 (2003).

4. Deepak Kundalkar, Asim Tewari. International Journal of Metallurgical Engineering. 2(2). P. 117-124 (2013).

5. D. Dossu, Z. Azari, G. Pluvinage. Problems of strength. 6. P. 32-42 (1998) (In Russian) [Д. Доссу, 3. Азари, Г. Плювинаж. Проблемы прочности. 6. С. 32-42 (1998)].

6. J. Gubicza, S.V. Dobatkin, E. Khosravi. Reduction of vacancy concentration during storage of severely deformed $\mathrm{Cu}$. Materials Science and Engineering A 527. (2010). P. 6102-6104.

7. V.M. Kosenkov. Letters on Materials. 4(1). 18 (2014) (in Russian) [B.M. Косенков. Письма о материалах. 4(1). 18 (2014)].

8. G.Kolskiy. Solid bodies strain waves. Moscow, Izdatelstvo inostrannoy literatury. (1955). 195 p. (in Russian) [Г. Кольский Волны напряжения в твердых телах. Москва, Изд-во иностр. лит. 1955. 195 с].

9. A.M. Bragov, A.K. Lomunov. Applied problems of strength and plasticity: All-Union interacademic collection of Nizhegorodskiy University. (1995). 51. P. 127-137 (in Russian) [A.M. Брагов, А.К. Ломунов. Прикладные проблемы прочности и пластичности: Всесоюз. межвуз. сб. Нижегородского ун-та. 1995. 51. C. 127-137].

10. V.M. Kosenkov, V.M. Bychkov. Applied Mechanics and Technical Physics. 53(6). P.134-143 (2012). (In Russian) [В.М. Косенков, В.М. Бычков. ПМТФ. 53(6). С.134143 (2012)], DOI: 10.1134/S0021894412060156

11. V.M. Kosenkov. Journal of Applied Mechanics and Technical Physics. 55(4). P. 33-42 (2014) (in Russian) [В.М. Косенков. ПМТФ. 55(4). С.33-42 (2014)].

12. T. Nicholas. Exp. Mech. 21(5). P. 177-185 (1981).

13. C. Salisbury, M.J. Worswick, R. Mayer. Journal de Physique. 134(4), P.43-48 (2006).

14. V.M. Kosenkov. Electronic material processing. 50(2), 81 (2014). (In Russian) [В.М. Косенков Электронная обработка материалов. 50(2), 81 (2014)].

15. H. Shen, C.J. Lissenden. Materials Science and Engineering. 8, P. 271-281 (2002).

16. M.A. Meyers. Dynamic Behavior of Materials. New York: John Wiley and Sons, Inc., (1994). 668 p. 